\title{
Macroeconomic Switching Regimes and Monetary Policy in Canada
}

\author{
Ronald Henry Lange \\ Correspondence: Ronald Henry Lange, Economics Department, Laurentian University, Sudbury, Ontario, P3E 2C6, \\ Canada.
}

Received: April 9, 2017

Accepted: May 7, 2017

Available online: June 1, 2017

doi:10.11114/aef.v4i4.2342

URL: https://doi.org/10.11114/aef.v4i4.2342

\begin{abstract}
This study examines the behaviour of monetary policy in Canada over the last 40 years using a Markov-switching VAR model of the macroeconomy. The Markov-switching estimates capture three continuous regimes that are interpreted as the 'surprise' regime from 1972Q1 to 1982Q2, the 'recovery' regime from 1982Q3 to 1991Q3 and the 'target' regime from 1991Q4 to 2014Q4. Monetary policy multipliers for the output gap are greater than one for all three regimes, suggesting that the central bank does not accommodate any expected changes in inflation over the long-run due to the domestic relationship between the output gap and future inflation. The long-run multipliers for inflation are equal to one in the surprise and recovery regimes, indicating that monetary policy also responds to offset inflation shocks. Overall, the policy multipliers and impulse response functions indicate a proactive central bank that responds systematically to movements in the output gap in order to control expected future inflation and to inflation surprises in the three regimes. The regime-dependent behaviour of monetary policy indicates a central bank pursuing an implicit form of inflation targeting as a means of achieving a nominal anchor for policy. The implicit inflation targets are consistent with historical episodes of inflation in Canada over the past 40 years.
\end{abstract}

JEL Classification: E44, E52

Keywords: monetary policy, policy multipliers, inflation targets, regime switching, vector autoregessions

\section{Introduction}

In February 1991, the Bank of Canada and the Government of Canada jointly announced explicit targets for reducing the rate of inflation on a path to price stability and adopted an inflation target of 2 percent inflation for the consumer price index. The announcement followed the pioneering move by the Bank of New Zealand in 1990. Since then almost 25 countries have adopted inflation targets, including the United States where the Federal Reserve in 2008 adopted an official objective of 2 percent for the deflator for personal consumption expenditures. The now widespread adoption of explicit inflation targets poses at least two questions and hypotheses about the behavior of monetary policy. First, what did central banks use as a nominal anchor for monetary policy in previous periods? One hypothesis is that they also targeted inflation, but as an implicit goal. Second, has there been a notable change in the behavior of monetary policy since the adoption of explicit inflation targets? Another hypothesis is that monetary policy has become more proactive in responding to expected future inflation since the adoption of explicit inflation targets. These questions and hypotheses are addressed in this study of macroeconomic switching regimes in Canada.

This study uses a Markov-switching vector autoregression (MS-VAR) with regime-dependent dynamics to study the systematic monetary policy responses to output and inflation and to derive implicit inflation targets in Canada. The regime-switching methodology is particularly attractive for studying the evolution of monetary policy because it allows for changes in behaviour in response to movements in the intermediate macroeconomic variables that are important for policy. The VAR approach has been criticized because of its limitations to identify the systematic part of monetary policy, leaving just a reaction function in surprises (Clarida, Gali, \& Gertler, 2000). However, this study allows for changes in the systematic behaviour of monetary policy to unexpected shifts in the processes of macroeconomic fluctuations in each regime. Monetary policy multipliers and impulse responses functions are calculated to capture the systematic responses to exogenous disturbances in inflation and the output gap. The study also contributes to the ongoing research that uses regime-switching models to study the behaviour of monetary policy by estimating implicit inflation targets for the macroeconomic regimes.

The main contribution of this study is the identification of three continuous stochastic regimes for the Canadian macroeconomy and the extraction of inflation targets for these regimes. The first regime is interpreted as a 'surprise' 
regime with an implicit inflation target of almost 8.0 per cent, reflecting the huge run-up in inflation following the unexpected huge oil-price shocks in the 1970s and early-1980s. The second is a 'recovery' regime with an implicit inflation target of about 4.5 per cent, where inflation is brought down to its level in the five years before the surge in inflation. The third is the 'target' regime with an explicit inflation target of 2.0 per cent, the mid-point of the target range adopted by the Bank and Government of Canada in 1991. The characteristics and properties of the macroeconomic switching regimes and the regime-dependent behaviour of monetary policy with respect to the output gap and inflation in the three regimes are analyzed. The implicit and explicit forms of inflation targeting suggest a central bank pursuing inflation targets as a nominal anchor for monetary policy, consistent with the first hypothesis mentioned above.

The following section discusses some recent literature on monetary policy in the context of macroeconomic switching regimes. Section 3 outlines the Markov-switching (MS) technique for the multivariate (VAR) estimations and discusses the data and the preliminary specification of the MS-VAR model. Section 4 presents the estimation results for the 3-regime specification of the model and discusses the characteristics and properties of the regimes. Section 5 derives long-run policy multipliers and impulse response functions that characterize the systematic responses of monetary policy and estimates implicit inflation targets for monetary policy in the regimes. The final section discusses the implication of the results for future monetary policy and research.

\section{Literature Review}

\subsection{Regime-switching Vector Autoregression Studies}

Since the seminal work by Hamilton (1989) on U.S. business cycles, the Markov-switching technique been widely applied in various scenarios. The earlier applications were with autoregessive (AR) models. However, more recently, the technique has been applied to the simultaneous equation framework by using vector autoregessions (VAR) to study interest rates (e.g., Ang \& Bekaert, 2002), exchange rates (e.g. Chen, 2006 and Soledad \& Peria, 2002), and international and regional business cycles (e.g., Krolzig, 2001 and Krolzig \&Toro, 2005), as well as other areas.

The comprehensive study by Sims and Zha (2006) is particularly important because it establishes a baseline for empirical research on macroeconomic switching. In general, they allow residual variances and coefficients for all equations in the VAR to change between states in order to focus on nonlinearities that lead to a deterioration of goodness of fit and heteroskedasticity. They find that their best fit is the one that allows for no time variation in the coefficients of the policy rule or the private sector block of the model, but allows for the disturbance variances of the model to change among three states. Their best-fitting model among those that allow coefficients to change is one that constrains the changes to occur in the monetary policy equation, while coefficients in the other equations remain constant. In general, they find more evidence of a stable transmission mechanism with unstable disturbance variances than of clear changes in model dynamics.

Rubio-Ramírez, Waggoner, and Zha (2005) find similar evidence for the European Monetary Union (EMU) using various Markov-switching structural VAR models (MS-SVAR) to analyse whether monetary policy and the volatility of euro-area macroeconomic variables have changed since the introduction of the EMU in 1993. They find that the source of time variation embedded in euro area aggregate variables can be attributed mainly to changes in shock variances. Similar to Sims and Zha (2006), they find that the MS-SVAR models based solely on time-varying shock variances are strongly favoured to models in which slope coefficients are also allowed to change with regimes. They find a stable and persistent post-1993 regime that is associated with low volatility of shocks to output, prices and interest rates, and that the output effects of monetary policy shocks are small and uncertain across regimes and models. These results are robust to various identification schemes for monetary policy.

However, some recent studies find that state-dependent dynamics are relatively important for capturing macroeconomic dynamics. Paolillo and Petragallo (2004), for example, use the MS-VAR methodology to allow for state-dependent, impulse response functions in order to analyse possible asymmetries in the business cycle transmission between US and Euro area. In short, they attempt to establish whether there is a transmission channel that depends on the state of the economy (low and high growth). They find strong evidence in favour of asymmetries in the transmission mechanism of the business cycle due to the interest rate differential and the Euro dollar exchange rate.

A number of recent studies have also found that state-dependent dynamics in regime-switching VARS are particularly useful for identifying structural changes in the monetary transmission mechanisms. Gonzalez and Garcia (2006) found that there was a major structural change in the transmission mechanism of monetary policy in Mexico around the beginning of 2001, the date of the formal adoption of the inflation targeting framework for the conduct of monetary policy. They found that this structural change implied a less important role for fluctuations of the real exchange rate in the process of price formation and in the formation of inflation expectations, as well as a milder effect on the nominal interest rate. The adoption of the inflation targeting framework in Mexico involved a stronger reaction of the nominal 
interest rate to increases in the output gap and the inflation rate and that the nominal interest rate had a stronger effect on the real exchange rate.

Similarly, Fujiwara $(2003,2006)$ uses a MS-VAR with regime-dependent, impulse-response functions to study whether the effects of monetary policy have changed during 1990s and whether the zero bound of nominal interest rate may have had some distortional effects on macroeconomic dynamics in Japan. The regime-dependent impulse response functions in these studies suggest that there was a structural change in the 1990s and that the traditional interest-rate channel was not functioning and, therefore, the role of monetary expansion is now limited. However, Girardin and Moussa (2009) used a factor-augmented, Markov-switching VAR (MS- FAVAR) that included the information in 143 variables for factors on real activity, prices, the monetary base, and interest rates in Japan. They find that the regime-dependent response of the output factor is three times as large in the post-1995 regime as in the pre-1995 regime and that it is 50 per cent longer-lived. The response of the price factor, while slightly smaller, is much longer-lived (up to nine months) than in the first regime. Overall, the non-neutrality of money and the price divergence in the pre-1995 regime that characterized the MS-VAR model in Fujiwara (2006) disappeared with the contribution of the information contained in the additional factors.

Lange (2016) also employed a Markov-switching VAR with regime-dependent dynamics to assess the transmission mechanism of monetary policy before and after the introduction of explicit-inflation targeting in Canada in 1991. The empirical results indicate relatively large differences in both the innovation process due to the variance component of the VAR and the propagation mechanism due to the regime-dependent systematic component. The pre-targeting regime corresponds to much larger innovations in the macroeconomic variables and overall larger systematic responses of the macroeconomic variables to the innovation processes, suggesting a stronger monetary transmission mechanism in the pre-target regime. However, variance decompositions and counterfactual analysis suggest that monetary policy has become more responsive to fluctuations in output growth and inflation in the target regime.

Also, more recently, Hubrich and Tetlow (2015) use a five variable MS-VAR for the U.S. with personal consumption expenditures, core CPI inflation, the federal funds rate, M2 growth, and a financial stress index to show that both variance and coefficient switching are needed to capture the linkage between financial stress and the macroeconomy. They show that shifts in the transmission mechanism of monetary policy during periods of financial stress coincide with the regime changes in the innovation processes.

\subsection{Monetary Policy Rules and Inflation Targets}

The second relevant strand of research for this study is the empirical analysis of monetary policy behaviour. Typically, the research focuses on some sort of an interest rate rule for monetary policy. Clarida, Gali and Gertler (1998) derive implicit inflation targets from policy reaction functions for the G3 (Germany, Japan, and the U.S.) and E3 (U.K., France, and Italy) countries. They find that inflation targets were about 2 per cent for Germany and Japan and about 4 per cent for the U.S. from 1979 to the early 1990s. Clarida et al. (2000) use a similar reaction function to derive inflation targets for the U.S. for periods corresponding to the tenures of various Federal Reserve chairs. They find that the inflation targets were about 4.25 per cent for pre-Volcker period (1960Q1-1979Q2) and about 3.60 per cent for the Volcker-Greenspan period (1979Q3-1996Q4). Both studies rely on estimates of single reaction equations for particular sample periods with instrumental variables from a generalized method of moments (GMM) estimation to handle simultaneity issues.

The shortcomings of the assumptions of linearity and time invariance in these Taylor-rule studies have been mitigated in some studies with Markov-switching equations to estimate monetary policy rules. For example, Dueker and Fischer (1996) estimated an equation of money growth for Switzerland with Markov-switching coefficients that allow for feedback from deviations in the price level and the exchange rate. Similarly, Dueker and Kim (1999) estimate a monetary policy feedback rule for Korea with switching coefficients for deviations in the price level and in industrial production. An obvious limitation of these studies is the use of single equation models that cannot be consistently estimated because the regressors are also endogenous. Valente (2003) overcomes the issues of linearity, simultaneity and time invariance by estimating a 2-regime switching VAR to derive implicit inflation targets for the G3 and E3 countries in Clarida et al. (1998).

\section{Methodology}

\subsection{Empirical Model}

The m-regime, $\mathrm{p}$-th order Markov-switching, vector autoregression for the multivariate estimations is the following intercept-switching, heteroskedastic, vector autoregression, or MSIH(m)-VAR(p) in the terminology of Krolzig (1997): ${ }^{1}$

\footnotetext{
${ }^{1}$ See Krolzig (1997) for a discussion of the Markov-switching technique for vector autoregression models of the business cycle.
} 


$$
\mathrm{y}_{\mathrm{t}}=v+\sum_{\mathrm{i}=1}^{\mathrm{p}} \mathrm{A}_{\mathrm{i}} \mathrm{y}_{\mathrm{t}-\mathrm{i}}+\varepsilon_{\mathrm{t}}
$$

where $y_{t}=\left[y_{1 t}, y_{2 t}, \ldots, y_{K t}\right]^{\prime}$ is a k-dimensional vector of observed time series for $t=1, \ldots \mathrm{T}$, $v=\left[v_{1}\left(s_{t}\right), v_{2}\left(s_{t}\right), \ldots, v_{K}\left(s_{t}\right)\right]^{\prime}$ is a k-dimensional column for regime-dependent intercept terms and $s_{t}$ is an unobserved discrete-regime variable evolving according to a time-varying, state-dependent process, the $\mathrm{A}_{\mathrm{i}}$ 's are a $\mathrm{k} \mathrm{x} \mathrm{k}$ matrix of autoregressive parameters, and $\varepsilon_{\mathrm{t}}=\left[\varepsilon_{1 \mathrm{t}}, \varepsilon_{2 \mathrm{t}}, \ldots, \varepsilon_{\mathrm{Kt}}\right]^{\prime}$ is a $\mathrm{k}$ - dimensional vector of Gaussian white noise processes for each regime i with covariance matrix $\Sigma$ conditioned on $s_{t}, \varepsilon \sim \mathrm{NID}\left(0, \Sigma\left(\mathrm{s}_{\mathrm{t}}\right)\right)$. In the MSIH-VAR model for the full sample, the autoregressive parameters are regime independent, consistent with the findings in the extensive study of macroeconomic switching by Sims and Zha (2006). However, since the estimated regimes in this study are continuous, the autoregressive parameters can be estimated separately for each regime to evaluate this hypothesis as will be discussed below.

For $m$ regimes, $s_{t} \in\{1, \ldots, m\}$, the matrix equation (1) may be summarized as

$$
\mathrm{y}_{\mathrm{t}}=\left[\begin{array}{c}
\mathrm{y}_{1 \mathrm{t}} \\
\cdots \\
\mathrm{y}_{\mathrm{mt}}
\end{array}\right]=\left[\begin{array}{c}
v_{1}+\sum_{\mathrm{j}}^{\mathrm{p}} \mathrm{A}_{1 \mathrm{j}} \mathrm{y}_{\mathrm{t}-\mathrm{j}}+\sum_{1}^{1 / 2} \varepsilon_{\mathrm{t}} \\
\cdots \\
v_{\mathrm{m}}+\sum_{\mathrm{j}}^{\mathrm{p}} \mathrm{A}_{1 \mathrm{j}} \mathrm{y}_{\mathrm{t}-\mathrm{j}}+\sum_{\mathrm{m}}^{1 / 2} \varepsilon_{\mathrm{t}}
\end{array}\right],
$$

where the intercepts $v_{\mathrm{i}}$ for $\mathrm{i}=1, \ldots, \mathrm{m}$ in equation (2) are simply the regime- weighted average of the means of the residuals from the VAR. Unlike regime-invariant models, the MSIH-VAR allows for shocks to the white-noise series $\varepsilon_{\mathrm{t}}$. The specification has the advantage of allowing the mean intercept to smoothly approach a new level after the transition from one state to another. ${ }^{2}$

The description of the data-generation process is not completed by the observational equation (1). A model for the regime-generating process is needed to allow for inference about the evolution of regimes from the data. The special characteristic of the Markov-switching model is the assumption that the unobservable realization of regime $\mathrm{s}_{\mathrm{t}} \in\{1, \ldots, \mathrm{m}\}$ is governed by a discrete-time, discrete-state Markov stochastic process. Formally, the stochastic process is defined by the transition probabilities

$$
P_{i j}=\operatorname{Pr}\left(s_{t+1}=j \mid s_{t}=i\right), \sum_{j=1}^{m} P_{i j}=1 \forall i, j \in\{1, \ldots, m\} .
$$

More specifically, $s_{t}$ is assumed to follow an ergodic and irreducible m-state Markov chain of order one with the transition matrix

$$
\mathrm{P}=\left[\begin{array}{cccc}
\mathrm{P}_{11} & \mathrm{P}_{12} & \ldots & \mathrm{P}_{1 \mathrm{~m}} \\
\mathrm{P}_{21} & \mathrm{P}_{22} & \ldots & \mathrm{P}_{2 \mathrm{~m}} \\
\ldots & \ldots & \ldots . & \ldots \\
\mathrm{P}_{\mathrm{m} 1} & \mathrm{P}_{\mathrm{m} 2} & \ldots & \mathrm{P}_{\mathrm{mm}}
\end{array}\right],
$$

where $p_{i m}=1-p_{i 1}-\cdots-p_{i, m-1}$ for $i=1, \ldots, m$. By inferring the probabilities of the unobserved regimes conditional on an available data set, it is then possible to reconstruct the regimes. For an ergodic Markov chain, regime shifts are persistent if $\mathrm{p}_{\mathrm{ij}} \neq \mathrm{p}_{\mathrm{ii}}$ for $\mathrm{i} \neq \mathrm{j}$ and not permanent if $\mathrm{P}_{\mathrm{ii}} \neq 1$ for all $\mathrm{i}$.

The two components of the MSIH(m)-VAR(p) model, the Gaussian multivariate model (1) as the conditional data generating process, and the Markov chain (3) as the regime generating process, are estimated using a likelihood-based statistical method. The maximization of the likelihood function of the MS-VAR entails an iterative technique to obtain estimates of the intercepts $v\left(\mathrm{~s}_{\mathrm{t}}\right)$ and variance-covariance matrices $\Sigma\left(\mathrm{s}_{\mathrm{t}}\right)$ and the transition probabilities $\mathrm{p}_{\mathrm{ij}}$ governing the Markov chain of the unobserved states. The maximum likelihood estimation (ML) is based on the implementation of the Expectation Maximization (EM) algorithm proposed by Hamilton (1990) for this class of models. Each iteration of the EM algorithm involves two steps. The expectation step involves a pass through the smoothing algorithm, using the estimated parameter vector for the VAR of the last maximization step in place of the unknown true parameter vector to infer the hidden Markov chain. In the maximization step, an estimate of the parameter vector is derived as a solution of the first-order conditions of the likelihood function, where the conditional regime probabilities are replaced with the smoothed probabilities derived in the last expectation step. These two steps are repeated until convergence is achieved for the maximum likelihood function.

\footnotetext{
${ }^{2}$ In contrast, a mean-switching, heteroskedastic, vector autoregessive model has a multiplicative relationship between the VAR coefficients and the intercepts that allows for an immediate one-time, permanent jump in the process mean after a change in regime. The specification is more appropriate for discrete switching variables, such as GDP in the studies of expansion and recession regimes.
} 


\subsection{Data and Preliminary Specification}

The estimation period is from 1972:1 to 2014:4 and the frequency is quarterly. ${ }^{3}$ The macroeconomic variables for the estimations include the overnight financing rate (ron) as the monetary policy rate, ${ }^{4}$ the output gap (gap) based on GDP in 2002 chain-linked dollars, the consumer price index (infl) for all items, and the change in the real exchange rate (pfx). The inflation rate is the log-difference over four quarters and scaled by 100 so that changes can be interpreted as the 4-quarter percentage change, consistent with the specification of the current inflation targets in Canada. ${ }^{5}$ The real exchange rate is defined as the Canada-US nominal exchange rate (e.g., price of a unit of US currency in terms of Canadian currency) multiplied by the ratio of the US GDP deflator to the Canadian GDP deflator. The real exchange rate is the log-difference over one quarter scaled by 100 so that changes can be interpreted as a 1-quarter percentage change. The output gap is defined as the log-difference of GDP and trend output scaled by 100, which builds in a demand/supply separation whereby potential output is the supply side and actual output the demand side.

The trend for output is calculated using a Hodrick-Prescott filter that minimizes the weighted sum of squared deviations from trend and the squared changes in the growth rate of the trend. This weighting is controlled by a smoothness parameter for the ratio of the variances set at 1600 , which is the common choice for quarterly data. The 2-sided HP filter has a well known problem of pulling the trend too close to the last data point of GDP, resulting in a closed output gap in 2014 when the economy reportedly had a negative output gap. As a standard fix to mitigate this problem, an ARIMA (1, 1,1) was used on the log of GDP to dynamically forecast out 12 quarters. The HP filter was then applied to the extend series, which shows a negative output gap mainly from 2008 to 2014, presented later in Figure 4. The four macroeconomic variables, the minimum needed for a small scale VAR of an open economy, are ordered as $\mathrm{Y}=$ [gap, infl, ron, pfx], so that inflation responds to the output gap in the current quarter, the monetary policy rate responds to both the output gap and inflation, and the exchange rate responds to all variables. The choice of macroeconomic variables is based on the view that the primary objective of monetary policy in an open economy like Canada is to control future inflation by responding to movements in the output gap that relates to future inflation in a Phillips-curve relationship and by reacting to inflation surprises that may be due to external shocks, such as shocks to the prices of oil and other commodities.

Augmented Dickey-Fuller tests for a unit root easily reject non-stationarity for the output gap and the real exchange rate at the 95 per cent level, while non-stationary cannot be rejected for the overnight policy rate and for the 4-quarter percentage change in the CPI for the sample period beginning in 1972. The null of a unit root in either variable is hard to reject at conventional significance levels because of the persistence of both series and the well-know low power of unit root tests. However, the non-stationary results appear to be mainly an artifact of the short estimation period and may reflect economic disequilibrium or the lack of a constant nominal anchor over part of the estimation period, especially during the 1970s and early- 1980s. ${ }^{6}$ In fact, non stationary can be rejected at the 95 per cent level for both series on a shorter sample period beginning in 1982 .

An issue of paramount difficulty in specifying Markov-switching models is the choice of the number of regimes. The likelihood-ratio test statistic for testing the number of regimes does not possess an asymptotic $\chi^{2}$ distribution because of the existence of a nuisance parameter under the null hypothesis. This study uses the general-to-specific approach to econometric modelling proposed by Krolzig (1997). A univariate ARMA analysis was performed on each of the macroeconomic time series. The test procedure relies on the following theorem: in the class of MSIH-AR models, there exists for any ARMA $\left(p^{*}, q^{*}\right)$ representation with $p \geq q \geq 1$ a unique MSIH (m)-AR (p) with $m=q^{*}+1$ and $p=p^{*}-q^{*} \cdot 7$ The Akaike information (AIC) and the Schwartz criteria (SC) are employed to assist in choosing the appropriate order of the ARMA (p, q) processes, which enables selection of the MSIH-AR models that can be expected to be consistent with the data.

Table 1 presents the results for a selection of ARMA models with 2 to 4 regimes and at least 3 lags. Generally, the results are quite mixed with the test procedure choosing 3 regimes for the overnight rate, 4 regimes for the inflation rate, and 2 or 3 regimes for the output gap, and 3 regimes for the change in the real exchange rate. Similarly, the test

\footnotetext{
${ }^{3}$ The beginning of the sample coincides with the introduction of flexible exchange rates.

${ }^{4}$ Armour et al. (1996) find that innovations in the overnight rate, derived using a Choleski decomposition, were consistent with intended policy actions as described in the Bank of Canada's Annual Reports since the early 1960s.

${ }^{5}$ The 4-quarter specification is also preferred because the consumer price index for Canada is not seasonally adjusted going back to 1972 .

${ }^{6}$ The assumption of a random walk may also not apply if a lower bound exists at low levels of interest rates, which has been the situation over the last few years of the sample period.

${ }^{7}$ See Krolzig (1997, p.130) for the theorem.
} 
procedure regarding the lag lengths are mixed, supporting either 3 or 4 lags. Preliminary estimations with univariate time series indicated that 3 regimes are most appropriate for the overnight rate and the inflation rate, consistent with the findings of Sims and Zha (2006) for the U.S.

Table 1. ARMA representation based model pre-selection for univariate specification

\begin{tabular}{|c|c|c|c|c|c|c|c|c|c|}
\hline \multirow[t]{2}{*}{$\operatorname{ARMA}(p, q)$} & \multicolumn{2}{|c|}{ policy } & \multicolumn{2}{|c|}{ inflation } & \multicolumn{2}{|c|}{ output gap } & \multicolumn{3}{|c|}{ exchange rateMSI $(m)-A R(p)$} \\
\hline & SIC & AIC & SIC & AIC & SIC & AIC & SIC & AIC & \\
\hline $\operatorname{ARMA}(4,1)$ & 3.20 & 3.30 & 1.97 & 1.87 & 1.88 & 1.78 & -4.31 & -4.41 & $\operatorname{MSI}(2)-\operatorname{AR}(3)$ \\
\hline $\operatorname{ARMA}(5,1)$ & 3.21 & 3.32 & 1.91 & 1.79 & 2.07 & 1.96 & -4.29 & -4.40 & $\operatorname{MSI}(2)-\operatorname{AR}(4)$ \\
\hline $\operatorname{ARMA}(5,2)$ & 3.11 & 3.94 & 1.98 & 1.85 & 2.01 & 1.88 & -4.26 & -4.39 & $\operatorname{MSI}(3)-\operatorname{AR}(3)$ \\
\hline $\operatorname{ARMA}(6,2)$ & 3.00 & 2.84 & 1.83 & 1.90 & 2.00 & 1.84 & -4.32 & -4.47 & $\operatorname{MSI}(3)-\mathrm{AR}(4)$ \\
\hline $\operatorname{ARMA}(6,3)$ & 3.26 & 3.09 & 1.90 & 1.73 & 1.90 & 1.73 & -4.20 & -4.38 & $\operatorname{MSI}(4)-\operatorname{AR}(3)$ \\
\hline $\operatorname{ARMA}(7,3)$ & 3.37 & 3.18 & 1.82 & 1.63 & 1.94 & 1.75 & -4.18 & -4.37 & $\operatorname{MSI}(4)-\mathrm{AR}(4)$ \\
\hline
\end{tabular}

Notes: SIC is Schwartz information criterion, AIC is Akaike information criterion, and bold is for lowest values.

The choice of lag length for the VAR was also guided by the Akaike Information, Schwarz Bayesian and Hannan-Quinn Criteria, and sequential likelihood-ratio tests from 1 to 4 lags presented in Table 2, as well as the significance of the intercepts and lags. The likelihood-ratio tests choose 3 lags and the information criteria choose 2, 1 and 2 lags, respectively. However, the ARMA test procedure in Table 1 suggests 4 lags for the overnight policy rate, the inflation rate and the change in the real exchange rate. The estimation presented below is based on 4 lags because the (own) parameters for the fourth lag were significant at the 5 per cent level in three of the equations and a few of the important intercept coefficients were more highly significant than with three lags.

Table 2. Tests of lag length

\begin{tabular}{lllll}
\hline lag & AIC & SBC & HQC & LR test \\
\hline 1 & 2.80 & $\mathbf{3 . 1 8}$ & 529.9 & $\ldots$ \\
2 & $\mathbf{2 . 6 1}$ & 3.26 & $\mathbf{5 1 6 . 9}$ & 0.00 \\
3 & 2.64 & 3.57 & 544.7 & 0.01 \\
4 & 2.73 & 3.92 & 574.2 & $\mathbf{0 . 0 8}$
\end{tabular}

Notes: AIC is the Akaike Information Criterion, SBC is Schwarz Bayesian Criterion, HQC is the Hannan-Quinn Criterion, LR test is the p-values for sequential likelihood ratio tests, and bold is for lowest values for AIC, SBC and HQC and for significance at the 5\% level for the LR test.

\section{Macroeconomic Switching Results}

The MSIH(3)-VAR(4) model requires the estimation of a 4x4 vector with 64 autoregressive parameters, a column of 4 intercept terms for each of the 3 regimes, a matrix of 4 variances and 6 covariances for each regime, and 6 independent transition probabilities, for a total of 112 parameters. Since the likelihood function for regime-switching models is well-known to have a large number of local maximums in finite samples, model selection for the parameter estimates is for the highest (mean) log-likelihood value. The estimations were repeated with various starting values for the intercept parameters until the highest likelihood value was obtained. ${ }^{8}$

\subsection{Multivariate Estimations}

The results from the multivariate estimation of the VAR model are presented in Table 3. The t-statistics for the coefficients in parenthesis are computed from the standard errors of the log- likelihood at the maximum. Both intercept terms (regime-weighted average of the means of the residuals) and variances for the overnight policy rate (ron) indicate three distinct regimes in terms of means and variances: low-, medium- and high-regimes. The estimates for the

\footnotetext{
${ }^{8}$ In particular, the starting values used for the MSIH(3)-VAR(4) were based on small changes in the regime-switching intercepts from univariate estimations. The log-likelihood function presented is by far the largest estimated. The convergence criteria was $10^{-5}$ for all estimations.
} 
conditional variances of the policy rate reveal considerable differences in the levels of within-regime volatility between the low- and high- variance regimes, confirming the well-known evidence of heteroskedasticity of short-term interest rates.

Similarly, there is a pattern of progressively higher intercept terms across regimes for inflation and the output gap. The pattern of progressively higher variances is also observed for the output gap, but not the inflation rate, where variances in regime 1 and 3 are about the same and about twice as large as in regime 2. The estimates for intercepts and variances for the exchange rate do not indicate specific regime behaviour, where the variances are about the same in regimes 2 and 3 and slightly smaller than in regime 1. Overall, the regime-switching estimation captures conditional heteroskedasticity in the form of Markov switching in the scale of the variances, where the error terms of the equations for the policy rate and the output gap switch discretely among low-, medium- and high-variance regimes.

Table 3. Markov-switching-intercept-heteroscedastic estimates for the MSIH (3)-VAR (4) model

\begin{tabular}{lllll}
\hline & policy rate & inflation & output gap & exchange rate \\
\hline \multirow{2}{*}{$v_{1}$} & 0.27 & 0.31 & 0.13 & -0.49 \\
& $(2.18)$ & $(3.50)$ & $(1.66)$ & $(-1.37)$ \\
$v_{2}$ & 0.83 & 0.70 & 0.42 & -1.71 \\
& $(2.63)$ & $(3.61)$ & $(1.94)$ & $(-3.00)$ \\
$v_{3}$ & 1.44 & 1.84 & 0.70 & -0.83 \\
& $(2.63)$ & $(6.64)$ & $(2.29)$ & $(-1.26)$ \\
$\sigma_{1}^{2}$ & 0.27 & 0.32 & 0.15 & 0.10 \\
& $(7.02)$ & $(7.00)$ & $(6.60)$ & $(7.88)$ \\
$\sigma_{2}^{2}$ & 0.66 & 0.16 & 0.46 & 0.02 \\
& $(3.99)$ & $(4.22)$ & $(4.37)$ & $(4.08)$ \\
$\sigma_{3}^{2}$ & 2.68 & 0.37 & 0.70 & 0.02 \\
& $(5.18)$ & $(4.38)$ & $(5.06)$ & $(4.35)$ \\
$\bar{\rho}$ & 0.93 & 0.75 & 0.81 & 0.40 \\
& $(0.00)$ & $(0.00)$ & $(0.00)$ & $(0.00)$ \\
\hline
\end{tabular}

Notes: $v_{\mathrm{i}}$ is the mean intercept for regime $i, \sigma_{i}^{2}$ is the variance for regime $i$, and $\bar{\rho}$ is the sum of autoregressive coefficients. Coefficients for intercepts and variances for the change in the real exchange rate have been scaled by 100 for presentation purposes. Figures in parentheses for the intercepts and variances are t-statistics based on standard errors of the maximum function value and for the autoregressive coefficients are p-values for a Chi-square test on the sum of the coefficients.

\subsection{Macroeconomic Regimes}

Figure 1 presents the probabilities for the multivariate estimation of the macroeconomy. The Markov-switching estimates for the VAR stochastically divide the sample period into three continuous regimes, with the low-mean and -variance regime 1 beginning in 1991:4, the medium-mean and-variance regime 2 covering the period from 1982:2 until 1991:3, and the high-mean and -variance regime 3 covering the first 12 years of the sample period. The division is stochastic in the sense that the last two regimes could occur again. The characteristics and properties of the regimes are discussed below.
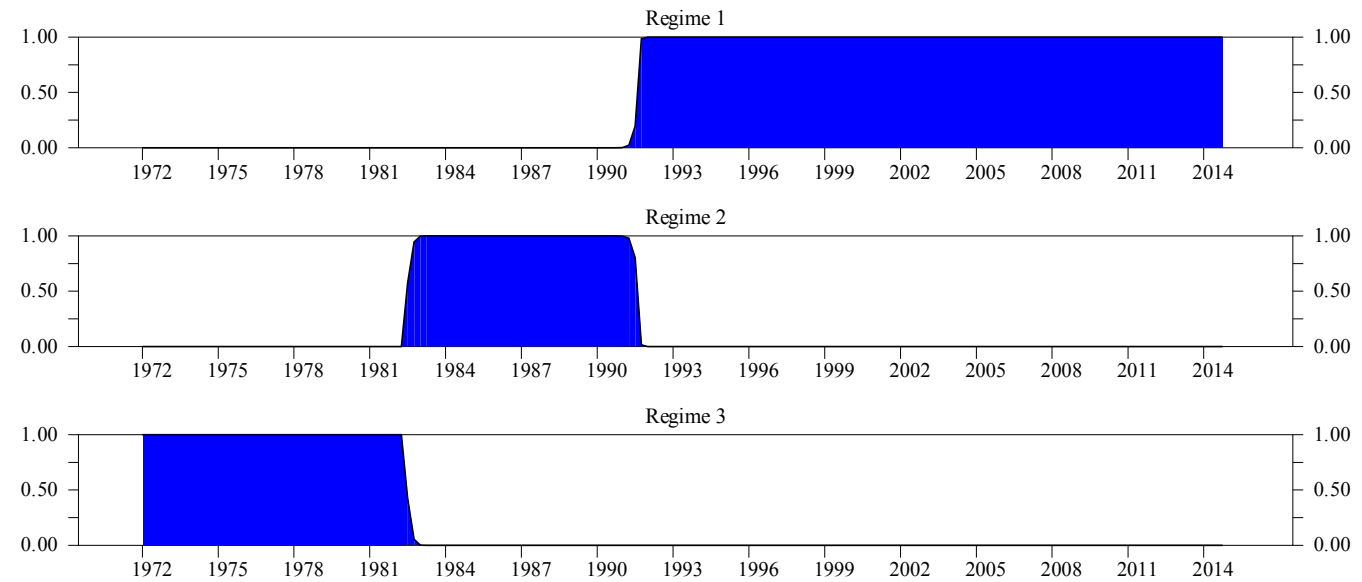

Figure 1. Macroeconomic switching regimes

Figure1 indicates that the regimes are very well classified with probabilities being relatively close to zero or one. The quality of the regime classification may be confirmed by the following the regime classification measure (RCM) proposed by Ang and Bekaert (2002) for m states

$$
\mathrm{RCM}=100 \mathrm{~K}^{2} \frac{1}{\mathrm{~T}} \sum_{\mathrm{t}=1}^{\mathrm{T}}\left(\prod_{\mathrm{i}=1}^{\mathrm{m}} \mathrm{p}_{\mathrm{i}, \mathrm{t}}\right),
$$


where $p_{i, t}=p\left(s_{t}=i \mid Y_{T}\right)$ is the unconditional probability of being in regime $i$. The constant 900 for three regimes serves to normalize the statistic to be between 0 and 100. A good regime classification is associated with low RCM values, with zero being a perfect classification and 100 implying that no information is revealed about the regimes. The RCM for the VAR regime-switching estimation presented in Figure 1 is virtually equal to zero, indicating that the regimes are perfectly classified. ${ }^{9}$

\subsection{Regime Characteristics}

Typical measures of the persistence of regimes are the probabilities of transition from one regime to another and the expected number of quarters in the regimes (duration). The estimated probabilities for the transition matrix for equation (4) are:

$$
P=\left[\begin{array}{lll}
0.994 & 0.030 & 0.000 \\
0.000 & 0.970 & 0.009 \\
0.006 & 0.000 & 0.991
\end{array}\right],
$$

where $P_{i j}=\operatorname{Pr}\left(s_{t+1}=j \mid s_{t}=i\right)$. Since all of the transition probabilities are below one, there are no permanent regimes. However, all regimes are very persistent, with probabilities of remaining in a regime $P_{i i}$ of at least 97 per cent, consistent with the virtually continuous regimes in Figure 1. There are only small probabilities of moving from one regime to another, with a probability of about 3 per cent for moving from the low-variance regime 1 to the medium-variance regime 2 and a probability of close to 1 per cent for moving from regime 2 to the high-variance regime 3 . There is no probability moving from regime 2 to regime 1 , but a very small probability of jumping from regime 3 to regime 1 .

Table 4 indicates that the Canadian economy experience the low-variance period over 50 per cent of the time over the sample period with duration, calculated as $1 /\left(1-\mathrm{P}_{\mathrm{ii}}\right)$ of over 41 years. Similarly, the economy experienced the high-variance period about 25 per cent of the time with duration of about 28 years. However, the medium-variance regimes also occurred almost 20 per cent of the time, but its duration is only $81 / 4$ years, reflecting the lower $\mathrm{P}_{\mathrm{ii}}$ of 0.97 in the P matrix above.

Table 4. Macroeconomic regime properties

\begin{tabular}{llll}
\hline Regime & Observations & Ergodic Probabilities & Duration \\
\hline 1 & 93 & 0.541 & 166.7 \\
2 & 37 & 0.215 & 33.3 \\
3 & 42 & 0.244 & 111.1 \\
\hline
\end{tabular}

Notes: Observations is the number of observations in regime $\mathrm{i}$ with $\mathrm{p}_{\mathrm{ii}}>0.5$ and duration is the expected duration of regime $\mathrm{i}\left(1 / 1-\mathrm{P}_{\mathrm{ii}}\right)$ based on estimates using the EM algorithm.

\section{Monetary Policy}

\subsection{Interest Rate Rule}

Most central banks are typically viewed as setting the short-term interest rate taking into account the current and future states of the economy. Clarida et al. $(1998,2000)$, for example, specify a monetary policy rule where central banks choose their interest rate targets according to expected inflation and output. For a small open economy like Canada, the forward-looking monetary-policy rule may also include the change of the real exchange rate: ${ }^{10}$

$$
r_{t}^{\mathrm{T}}=\mathrm{r}^{\mathrm{e}}+\beta\left(\mathrm{E}_{\mathrm{t}} \pi_{\mathrm{t}+1}-\pi^{\mathrm{e}}\right)+\gamma\left(\mathrm{E}_{\mathrm{t}} \mathrm{y}_{\mathrm{t}+1}-\overline{\mathrm{y}}\right)+\phi \Delta \mathrm{e}_{\mathrm{t}},
$$

where $r_{t}^{T}$ is the current interest-rate target, $\mathrm{r}^{\mathrm{e}}$ is the long-run, equilibrium, nominal target for the interest rate when both inflation and output are at their desired levels and the change in the exchange rate is zero, $\pi^{\mathrm{e}}$ is the inflation target, $E_{t} \pi_{t+1}$ and $\mathrm{E}_{\mathrm{t}} \mathrm{y}_{\mathrm{t}+1}$ are expected inflation and output for period $\mathrm{t}+1$ respectively (conditional on the information set available at time $t), \bar{y}$ is potential output, and $\Delta e_{t}$ is the change in the real exchange rate. The underlying view of the economy in this formulation of the policy rule is that future inflation is determined by a Phillips-curve relationship and current inflation is affected by external factors, such as the price of oil and other commodities.

\footnotetext{
${ }^{9}$ The RCM statistic is essentially a sample estimate of its variance.

${ }^{10}$ Clarida et al. (1998), for example, suggest that lagged values of the exchange rate as possible variables in the bank's information set at the time it chooses the interest rate. Lange (2013) finds evidence of an important role for the real exchange rate in a contemporaneous policy-reaction function for Canada during certain periods.
} 
Assuming some interest rate smoothing behaviour on the part of the central bank so that the current interest rate partially adjusts to the target level:

$$
r_{t}=(1-\rho) r^{T}+\rho r_{t-1}+\eta_{t}
$$

where the central bank eliminates a fraction $(1-\rho)$ of the gap between its current target level and its past value in each period, and $\eta_{t}$ is a zero mean exogenous interest rate shock that allows for non-systematic or random policy reactions. The $\eta_{t}$ random component to policy has been stressed in the identified VAR research on monetary policy. ${ }^{11}$

Combining the reaction function (6) with the partial adjustment mechanism (7) yields the following interest rate rule:

$$
r_{t}=v+\rho r_{t-1}+(1-\rho)\left(\beta \pi_{t}+\gamma\left(y_{t}-\bar{y}\right)+\phi \Delta e_{t}\right)+\varepsilon_{t},
$$

where $\varepsilon_{t}=(1-\rho)\left[\beta\left(E_{t} \pi_{t+1}-\pi_{t}\right)+\gamma\left(E_{t} y_{t+1}-y_{t}\right)\right]+\eta_{t}$ is the linear combination of forecast errors for expected inflation and output and the exogenous policy shock, $v=(1-\rho)\left(\mathrm{r}^{\mathrm{e}}-\beta \pi^{\mathrm{e}}\right)$, and $\rho \in[0,1]$ captures the degree of smoothing of interest rate changes. Equation (8) is essentially a Taylor-like policy rule for an open economy with partial adjustment.

Equation (8) essentially maps the policy reaction function (6) and (7) to the reduced form of the interest rate equation in the MSIH (3)-VAR (p) in matrix equation (1), where the autoregressive coefficients for the interest rate equation are $A_{31}=(1-\bar{\rho}) \gamma, A_{32}=(1-\bar{\rho}) \beta, A_{33}=\bar{\rho}$ and $A_{34}=(1-\bar{\rho}) \phi$ for $\mathrm{k}=4$ in the recursive ordering of the VAR for $\mathrm{i}=1, \ldots, \mathrm{p}$, where the $\bar{\rho} \mathrm{s}$ are the sum of the lagged coefficients in the policy rule.

\subsection{Systematic Monetary Policy Responses}

The parameter estimates for the autoregressive coefficients from the VAR (p) portion of the model can be used to calculate systematic monetary policy reactions. Table 5 presents the sum of the coefficients and the multipliers for the full sample period and the three macroeconomic regimes in Figure 1. The sum of the own autoregressive coefficients for the overnight rate $\bar{\rho}$ at 0.90 for the full sample period indicates considerable persistence, suggesting inertia in the target rate for monetary policy $\mathrm{r}_{\mathrm{t}}^{\mathrm{T}}$ in the partial adjustment mechanism (7) with only about 10 per cent of a change $(1-\bar{\rho})$ taking place within the quarter of change. The estimate of the sum of the coefficients on the output gap $\bar{\gamma}$ in the policy equation is equal to 0.13 , indicating that the long-run policy multiplier for the output gap, $(\mu=\bar{\gamma} /(1-\bar{\rho}))$, is equal to 1.6. The estimate of the sum of the coefficients for inflation $\bar{\beta}$ in the equation for the overnight policy rate is equal to -0.05 and the long-run multiplier $(\mu=\bar{\beta} /(1-\bar{\rho}))$ is equal to -0.6 . However, the estimate of $\bar{\beta}$ is not significant at conventional levels due to the estimate in regime 1. Overall, the estimates of the monetary policy multipliers suggests that the Bank of Canada has not reacted directly and systematically to inflation over the full sample period, but instead has been proactive in fighting expected future inflation by reacting directly and systematically to movements the output gap, which affect inflation indirectly through a Phillip's curve type of relationship.

Table 5. Long-run static equilibrium multipliers

\begin{tabular}{lllll}
\hline & $\begin{array}{l}\text { Full Sample } \\
(1972: 1-2014: 4)\end{array}$ & $\begin{array}{l}\text { Regime 3 } \\
(1972: 1-1982: 2)\end{array}$ & $\begin{array}{l}\text { Regime 2 } \\
(1982: 3-1991: 3)\end{array}$ & $\begin{array}{l}\text { Regime 1 } \\
(1991: 4-2014: 4)\end{array}$ \\
\hline Number of Lags & \multicolumn{1}{c}{$(4$ lags $)$} & \multicolumn{1}{c}{$(1$ lag $)$} & (2 lags) & (2 lags $)$ \\
\hline Overnight rate & & & & \\
$\operatorname{ron}(\bar{\rho})$ & $0.92(0.00)$ & $0.69(0.00)$ & $0.56(0.00)$ & $0.94(0.00)$ \\
$\operatorname{gap}(\bar{\gamma})$ & $0.13(0.02)$ & $0.65(0.03)$ & $0.52(0.00)$ & $0.10(0.09)$ \\
$\operatorname{infl}(\bar{\beta})$ & $-0.05(0.41)$ & $0.30(0.11)$ & $0.45(0.01)$ & $-0.15(0.04)$ \\
$\mu_{\text {gap }}=\bar{\gamma} / 1-\bar{\rho}$ & 1.62 & 2.09 & 1.18 & 1.67 \\
$\mu_{\text {infl }}=\bar{\beta} / 1-\bar{\rho}$ & -0.63 & 0.97 & 1.02 & -2.50 \\
\hline
\end{tabular}

Notes: $\bar{\rho}=\rho_{1} \mathrm{~L}+\cdots+\rho_{\mathrm{n}} \mathrm{L}^{\mathrm{n}}$ corresponds to the sum of own autoregressive coefficients in the equation for the overnight policy rate, and similarly $\bar{\gamma}$ and $\bar{\beta}$ are the sum of the coefficients for the lags of the output gap and inflation, respectively, in the equation for the overnight rate. The p-values for Chi-square tests on the sum of the lagged coefficients are indicated in brackets. The multipliers $\mu$ are equal to $\bar{\beta}$ and $\bar{\gamma}$ divided by $(1-\bar{\rho})$.

Since the regime-switching esimates for the smoothed probabilites in Figure 1 indicate exact breaks for the three continuous regimes, it was possible to reestimate the policy VAR model for the individual regimes without having to allow for regime-switching autoregessive parameters in the estimation for the full sample. The parameter estimates for the autoregressive coefficients from the VAR (p) portion of the model can also be used to calculate the long-run

\footnotetext{
${ }^{11}$ See, for example, Bernanke and Mihov (1998).
} 
multipliers for monetary policy reactions to the output gap and inflation in each of the three regimes. The $\bar{\rho}$ for the overnight rate indicates considerably more persistence in regimes 3 at about 0.7 , so that about 30 per cent of a change $(1-\bar{\rho})$ took place within the quarter. In regime $2, \bar{\rho}$ equals 0.56 indicating that close to 50 per cent of the change in the target policy rate $r_{t}^{\mathrm{T}}$ occurred in the current quarter, suggesting that monetary policy became much more reactive in the 'recovery' regime after the inflation surprises in the previous regime. The value of 0.94 for $\bar{\rho}$ in the current target regime indicates the considerable inertia observed for the full sample, suggesting that Bank of Canada became more cautious about smoothing interest rates in the current regime with explicit inflation targets.

Since the long-run monetary policy multipliers for the output gap are greater than one for the full sample and in all three regimes, $\mu_{\text {gap }}>1$, the target overnight rate does not accommodate any expected changes in inflation over the long-run due to the domestic relationship between the output gap and future inflation, which is consistent with the existence of both implicit and explicit inflation targets. Monetary policy only responds to inflation shocks or surprises in theses regimes due to external factors, such as the prices of oil and other commodities. In fact, the long-run multipliers for the systematic policy responses to inflation in the first two regimes 3 and 2 are virtually equal to one, $\mu_{\text {infl }}=1$, indicating that monetary policy operated to offset shocks to inflation due to inflation surprises in these regimes. The impulse responses functions below indicate that monetary policy initially increases the policy rate in response to an inflation shock in regime 1. However, on balance the multiplier for inflation is negative in the regime 1, reflecting that monetary policy placed a negative weight on inflation because inflation was consistently in the lower portion of its 2-percent target range with an average rate of 1.91 per cent. This is consistent with the second hypothesis mentioned earlier that monetary policy has become more proactive in responding to expected future inflation by reacting aggressively to movements in the output gap.

\subsection{Policy-impulse Responses to Macroeconomic Shocks}

The impulse responses functions for monetary policy to innovations in the output gap and inflation for the full sample and the three regimes are presented in the panels of Figure 2.The confidence bands for the impulse responses are calculated as the fractiles 0.16 and 0.84 using Monte Carlo simulations to estimate the deviations of the response functions. ${ }^{12}$ The confidence bands are displayed around the mean of the drawn responses for the impulse response functions, where "significant" for a response means statistically different from zero, which corresponds to the case where the error confidence bands of the response function lie on one side of the $\mathrm{x}$-axis. In general, the impulse responses provide some insight into the dynamics of the systematic behaviour of monetary policy to unexpected shifts in the processes of macroeconomic fluctuations not depicted by the long-run multipliers presented in the previous section.

The impulse responses to a 1-standard error innovation in the output gap indicates that monetary policy responds in regime 3 by raising the overnight policy by almost 1.25 percent by the fourth quarter. The overall response lasts about $21 / 2$ years. On the other hand, the monetary policy rate in regime 2 is raised by only about 0.75 per cent by the second quarter, about one-half the increase as in regime 3 . The response in regime 1 is even smaller at about 0.3 per cent by the third quarter. Also, the responses in regime 2 and 1 only last about one-half as long as in regime 1 . Monetary policy responses to innovations in the inflation rate are about the same in the first two regimes, increasing by about 0.30 percent by the second or third quarters. The notable difference is the marginal, but significant, response in regime 1 of about 5 basis points in the first quarter. Overall, the dynamics from the impulse responses support the results from the systematic responses calculated by the long-run multipliers; that is, monetary policy has been forward-looking by reacting to expected inflation from innovations in the output gap, but reacts almost immediately to unexpected inflation or surprises due to external factors.
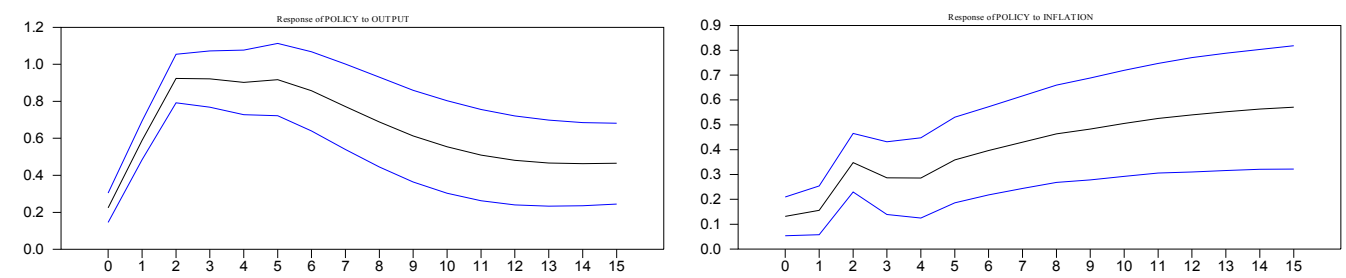

Full sample. 1972:1-2014:4

\footnotetext{
${ }^{12}$ Sims and Zha (1999) show that the use of 1- or 2-standard deviation bands can give a misleading impression because impulse responses often have a highly asymmetrical distribution. They recommend using fractiles instead of standard deviation bands.
} 

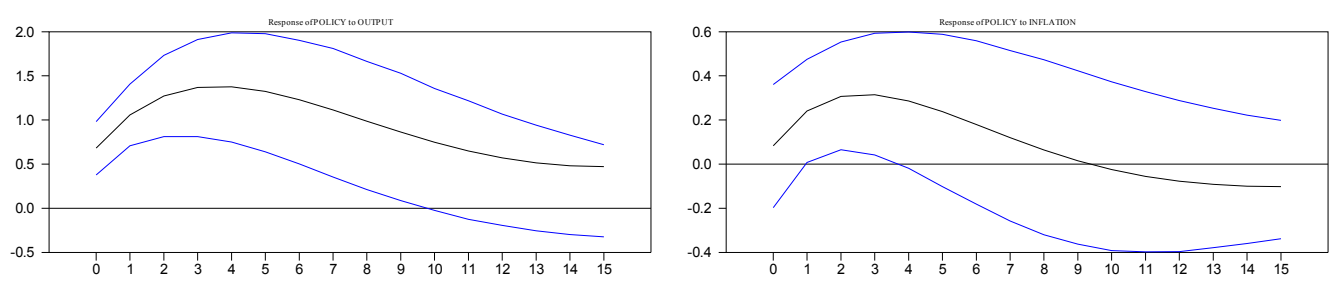

Regime 3: 1972:1-1982:2
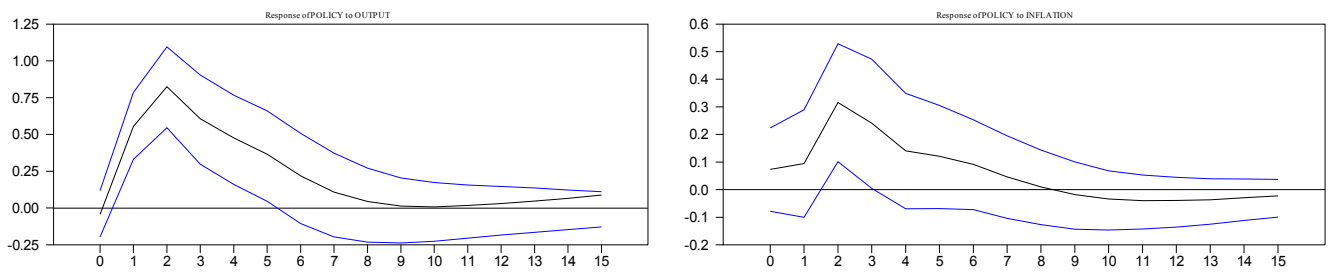

Regime 2: 1982:3-1991:3
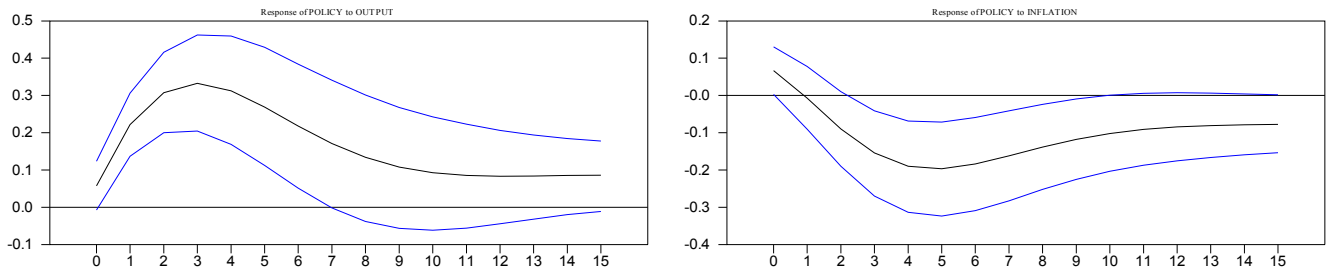

Regime 1: 1991:4-2014:4

Figure 2. Policy responses to 1-standard-error innovations in the output gap and inflation

\subsection{Implicit Inflation Targets}

The parameter estimates from the $\operatorname{MSIH}(3)$ portion of the interest rate equation in Table 3 indicate significant, sharp and persistent shifts in both the intercept terms and variances for all three regimes. In order to understand the possible source of the regime shifts, the intercepts $v$ for the three macroeconomic regimes can be used, along with the autoregressive parameters, to derive the implicit regime-inflation $\operatorname{targets} \pi^{\mathrm{e}}\left(\mathrm{s}_{\mathrm{t}}\right)$. Since in long-run equilibrium, the economy adjusts to potential output $\bar{y}$ and the change in the exchange rate $\Delta \mathrm{e}_{\mathrm{t}}$ is equal to zero, the interest rate and inflation rate equations are estimated as a bivariate VAR with only regime-switching intercepts. ${ }^{13}$

Only, the drift term $v=(1-\rho)\left(\mathrm{r}^{\mathrm{e}}-\beta \pi^{\mathrm{e}}\right)$ remains in interest rate rule (8) and can be rearranged to become:

$$
\pi^{\mathrm{e}}\left(\mathrm{s}_{\mathrm{t}}\right)=\left[\mathrm{rr}^{\mathrm{e}}-\left(v\left(\mathrm{~s}_{\mathrm{t}}\right) /(1-\bar{\rho})\right)\right](1 /(1-\bar{\beta}),
$$

where $\pi^{\mathrm{e}}\left(\mathrm{s}_{\mathrm{t}}\right)$ are the regime-dependent inflation targets, $\mathrm{rr}^{\mathrm{e}}=\mathrm{r}^{\mathrm{e}}-\pi^{\mathrm{e}}$ is the real interest rate target calculated with the Fisher relationship, $\bar{\rho}$ and $\bar{\beta}$ are the sum of the autoregressive coefficients for the overnight and inflation rates in the equation for the monetary policy rate, respectively, and $v\left(s_{t}\right)$ is a regime-switching drift term.

Although the empirical model does not separately identify $\mathrm{rr}^{\mathrm{e}}$ and $\pi^{\mathrm{e}}\left(\mathrm{s}_{\mathrm{t}}\right)$, equation (9) provides a relation between the two variables. Since the implied regime- switching inflation target $\pi^{\mathrm{e}}\left(\mathrm{s}_{\mathrm{t}}\right)$, is of more interest in the characterization of monetary policy, the long-run equilibrium real rate $\mathrm{rr}^{\mathrm{e}}$ is assumed to be determined by non-monetary factors and

\footnotetext{
${ }^{13}$ For the bivariate VAR, two lags were sufficient to eliminate any autocorrelation, consistent with the tests for lag length in Table 2.
} 
treated as constant across regimes. ${ }^{14}$ The average real rate $\overline{\mathrm{rr}}$ for the sample period of almost 40 years is used as an approximation of the long-run equilibrium real rate $\mathrm{rr}^{\mathrm{e}}$ for the overnight rate across regimes. ${ }^{15}$

The regime-dependent inflation targets and the inflation rate are presented in Table 6 and in Figure 3. The profiles of the inflation targets in Figure 3 mirror the macroeconomic switching regimes in Figure 2. ${ }^{16}$ The implicit target was almost 8.0 per cent until about 1982, drops down to about 4.5 per cent until about 1991, and then drops down to 2.00 per cent, the mid-point of the Bank of Canada's inflation target range. The two implicit target rates and one explicit target rate for inflation suggest that regime 3 represents a 'surprise' regime with high inflation due to the unexpected oil-price shocks in the 1970s, regime 2 represents a 'recovery' regime where inflation is brought down to its level close that before the huge run-up in inflation, ${ }^{17}$ and regime 1 represents the 'target' regime where the target is calibrated to match the mid-point of the explicit target range adopted by the Bank and Government of Canada. These inflation targets suggest a central bank pursuing inflation targets as a nominal anchor for monetary policy, consistent with the first hypothesis mentioned earlier.

Table 6. Regime-dependent inflation targets

\begin{tabular}{lccl}
\hline Regime & $\overline{\mathrm{rr}}$ & $\pi_{\mathrm{t}}$ & $\pi^{\mathrm{e}}$ \\
\hline 1 & 5.55 & 1.91 & 2.00 \\
2 & 5.55 & 4.81 & 4.58 \\
3 & 5.55 & 8.74 & 7.91 \\
Average & 5.55 & 4.16 & 3.92 \\
\hline
\end{tabular}

Notes: $\overline{\mathrm{rr}}$ and $\pi_{\mathrm{t}}$ and are the average real and inflation rates, respectively, and $\pi^{\mathrm{e}}$ is the inflation target. The average inflation target is weighted by the regime sample periods.

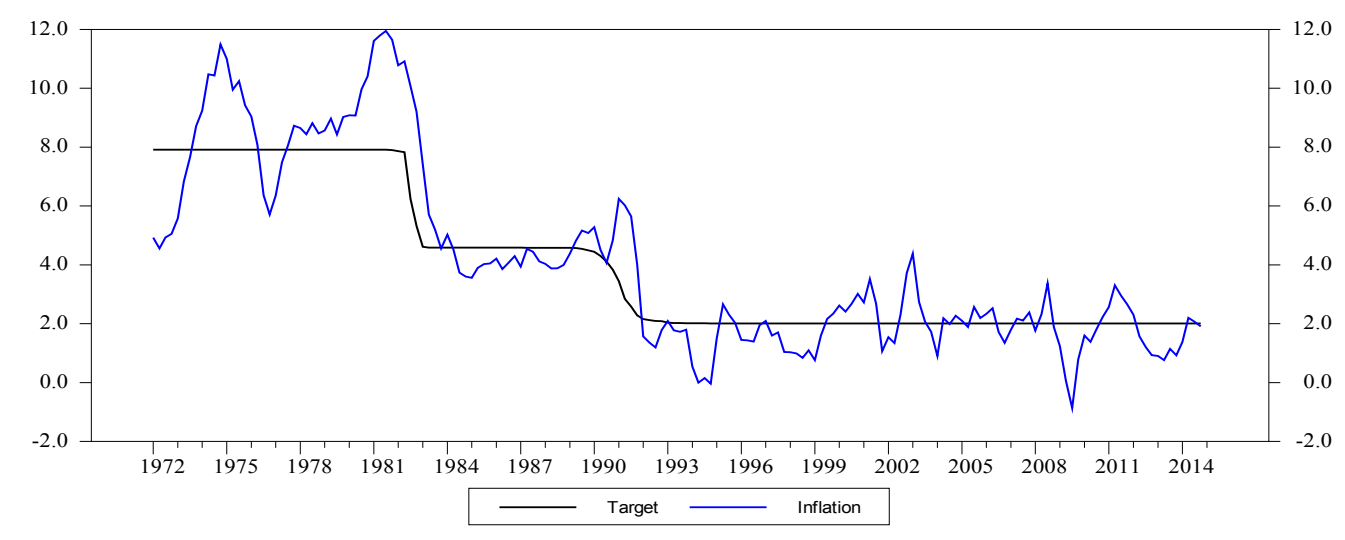

Figure 3. Inflation and implicit inflation targets

The inflation gap with the inflation targets in Figure 3 are presented in Figure 4, along with the output gap. The movements in the inflation gap generally lead those in the output gap by several quarters, as expected with a Phillip's-curve relationship between the output gap and inflation.

\footnotetext{
${ }^{14}$ The assumption of a constant real rate is also used by Clarida et al. (1998) and Valente (2003) to calculate implicit inflation targets. However, Clarida et al. (2000) calculate separate real rates for policy regimes corresponding to tenures of different Federal Reserve chairs.

${ }^{15}$ The estimate of the real overnight rate uses the quarterly inflation rate for the GDP deflator since a quarterly measure matches the frequency of the short-term nominal rate used as the monetary policy instrument, and the deflator is the broadest measure of inflation in the macroeconomy. However, the results are virtually identical using the quarterly change in the CPI.

${ }^{16}$ See the Appendix for a figure of the smooth probabilities from the MSI(3)-BVAR(2).

${ }^{17}$ The average inflation rate in the 5-year period prior to the first major oil-price shock in 1973 is 4.1 per cent.
} 


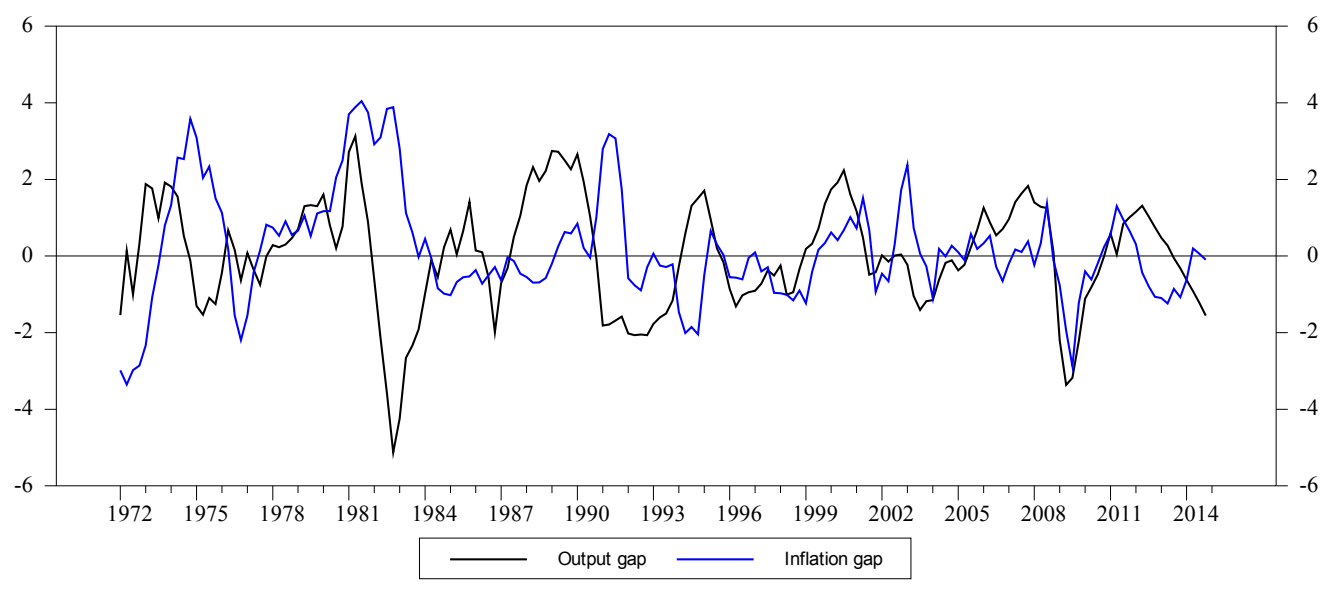

Figure 4. Output and inflation gaps

\section{Concluding Remarks}

This study estimates a MSIH(3)-VAR(4) model to examine the characteristics and properties of macroeconomic switching regimes in Canada and the regime-dependent behaviour of monetary policy. The estimation results identify three continuous regimes. The first regime is interpreted as a 'surprise' regime from 1972Q1 to 1982Q2 with an implicit inflation target of almost 8.0 per cent, reflecting the huge run-up in inflation following the unexpected large oil-price shocks in the 1970s. The second is a 'recovery' regime from 1982Q3 to 1991Q3 with an implicit inflation target of about 4.5 per cent, where inflation is brought down to near its level before the surge in inflation. The inflation target for the recovery regime in line with implicit inflation target of about 4 per cent estimated for the U.S. from 1979 to the early 1990s by Clarida et al. (1998). The third is the 'target' regime with an inflation target of 2.0 per cent, the mid-point of the explicit target range for inflation adopted by the Bank and Government of Canada in 1991. The calculated implicit and inflation targets for the three regimes of the sample period address the first question and hypothesis in the introduction; that is, they suggest a central bank pursuing inflation targets as a nominal anchor for monetary policy.

Long-run policy multipliers for the output gap are greater than one for the full sample and for all three regimes, indicating that the target overnight rate does not accommodate any expected changes in inflation over the long-run due to the domestic relationship between the output gap and future inflation, which is consistent with the existence of both implicit and explicit inflation targets for a forward-looking central bank. The long-run multipliers for inflation are equal to one in the surprise regime 3 and the recovery regime 2 , indicating that monetary policy also responded directly to completely offset inflation surprises in these regimes. The policy impulse response functions for the output gap and inflation also suggest systematic monetary policy reactions to movements in the output gap and to inflation shocks in all three regimes. The long-run multipliers for the output gap and inflation in the current regime with an explicit inflation target suggest a central bank reacting aggressively and proactively to movements in the output gap in order to limit expected future inflation so that it did not have to respond to surprise inflation shocks as it did in the previous two regimes. This addresses the second question and hypothesis posed in the introduction about the behaviour of monetary policy in the current target regime.

There are several implications of the estimation results for monetary policy in Canada. First, the monetary policy multipliers suggest a forward-looking central bank that responds aggressively to movements in the output gap in order to control future inflation. Second, the regime-switching estimation suggest a central bank pursuing an implicit form of inflation targeting before the adoption of an explicit inflation target as a means of gaining a nominal anchor for monetary policy. Third, the current macroeconomic regime with an explicit nominal target suggests a permanent shift to a new type of monetary policy behaviour. Finally, the current 'target' regime will be associated with lower volatility in interest rates, inflation and output in the future.

There are also a few implications of the estimation results for future research. The most obvious is the derivation of the implicit inflation targets for the last 40 year that can be use to incorporate an inflation gap directly into policy rules in models of the Canadian economy. Also, the estimation results in this study are based on a small scale open-economy VAR with only four macroeconomic variables. A larger model with other financial and economic variables that also affect monetary policy choices may provide richer insights into the behaviour of monetary policy in the current macroeconomic regime. For example, the recent macroeconomic-finance research by Diebold, Rudebusch and Aruoba 
(2006) suggest bidirectional links between the term structure of interest rates and the macroeconomy that may be important, especially for Canada ( see Lange, 2013). Also, the recent study on Japan by Kurihara (2017) investigates various augmented Taylor rules that suggests other macroeconomic variables may contain additional information for monetary policy decision making in addition to the typical output and inflation gaps.

\section{Acknowledgement}

The helpful comments of two anonymous referees are gratefully appreciated.

\section{References}

Ang, A., \& Bekaert, G. (2002). Regime switches in interest rates. The Journal of Business and Economics, 20, 163-182. https://doi.org/10.3386/w6508

Armour, J., Engert, W., \& Fung, B. (1996). Overnight innovations as a measure of monetary policy shocks in vector autoregressions. Bank of Canada Working Paper, 96-4. https://doi.org/10.2139/ssrn.56181

Bernanke, B., \& Mihov, I. (1998). Measuring monetary policy. The Quarterly Review of Economics, 113, 869-902. https://doi.org/10.3386/w5145

Chen, S. (2006). Revisiting the interest rate-exchange rate nexus: a Markov-switching approach. The Journal of Development Economics, 79, 208-224. https://doi.org/10.1016/j.jdeveco.2004.11.003

Clarida, R., Gali, J., \& Gertler, M. (1998). Monetary policy rules in practice: some international evidence. The European Economic Review, 42, 1033-1067. https://doi.org/10.1016/S0014-2921(98)00016-6

Clarida, R., Gali, J., \& Gertler, M. (2000). Monetary policy rules and macroeconomic stability: evidence and theory. The Quarterly Review of Economics, 115, 147-180. https://doi.org/10.1162/003355300554692

Diebold, F., Rudebusch, G., \& Aruoba, S. (2006). The macroeconomy and the yield curve: a dynamic latent factor approach. Journal of Econometrics, 131, 309-338. https://doi.org/10.1016/j.jeconom.2005.01.011

Dueker, M., \& Fischer, A. (1996). Interest targeting in a small open economy: empirical results for Switzerland. The Journal of Monetary Economics, 37, 89-103. https://doi.org/10.1016/0304-3932(95)01241-9

Dueker, M., \& Kim, K. (1999). A monetary policy feedback rule in Korea's fast-growing economy. The Journal of International Financial Markets, Institutions, and Money, 9, 19-31. https://doi.org/10.1016/S1042-4431(98)00034-1

Fujiwara, I. (2003). Has the effect of monetary policy changed during 1990s? Osaka School of International Public Policy, Discussion Paper 03-08.

Fujiwara, I. (2006). Evaluating monetary policy when nominal interest rates are almost zero. The Journal of the Japanese and International Economies, 20, 434-453. https://doi.org/10.1016/j.jjie.2006.02.001

Girardin, E., \& Moussa. Z. (2009). The effectiveness of quantitative easing in Japan: New evidence from a structural factor-augmented VAR, mimeo, http://www.edge-page.net/jamb2008/ Papers/MoussaEDGE2008.pdf

Gonzalez, A., \& Garcia, J. (2006). Structural changes in the transmission mechanism of monetary Policy in Mexico: A non-linear VAR approach. Bank of Mexico Working Paper 2006-06

Hamilton, J. (1989). A new approach to the econometric analysis of nonstationary times series and business cycles. Econometrica, 57, 357-384. https://doi.org/10.2307/1912559

Hamilton, J. (1990). Analysis of time series subject to change in regime. The Journal of Econometrics, 45, 39-70. https://doi.org/10.1016/0304-4076(90)90093-9

Hubrich, K., \& Tetlow, R. (2015). Financial stress and economic dynamics: The transmission of crises. Journal of Monetary Economics, 70, 100-115. https://doi.org/10.1016/j.jmoneco.2014.09.005

Krolzig, H. (1997). Markov Switching Vector Autoregressions: Modelling, Statistical Inference and Applications to Business Cycle Analysis. Berlin: Springer-Veriag. https://doi.org/10.1007/978-3-642-51684-9

Krolzig, H. (2001). Business cycle measurement in the presence of structural change: international evidence. The International Journal of Forecasting, 17, 349-368. https://doi.org/10.1016/S0169-2070(01)00099-1

Krolzig, H., \& Toro, J. (2005). Classical and modern business cycle measurement: the European case. The Spanish Economic Review, 7, 1-21. https://doi.org/10.1007/s10108-004-0088-0

Kurihara, Y. (2017). Taylor rule during the zero or low interest rate era: The recent Japanese case. Applied Economics and Finance, 4, 1-8. https://doi.org/10.11114/aef.v4i1.1909

Lange, R. (2013). Monetary policy reactions and the exchange rate: a regime-switching structural VAR for Canada. 
The International Review of Applied Economics, 27, 612-632. https://doi.org/10.1080/02692171.2012.752446

Lange, R. (2013). The Canadian macroeconomy and the yield curve: a dynamic latent factor approach. International Review of Economics and Finance, 27, 261-274. https://doi.org/10.1016/j.iref.2012.10.003

Lange, R. (2016). The monetary transmission mechanism and inflation targeting: A regime switching VAR approach for Canada. Applied Economics and Finance, 3, 263-279. https://doi.org/10.11114/aef.v3i2.1338

Paolillo, R. M., \& Petragallo, N. (2004). Asymmetries of monetary policy transmission between US and Euro area, mimeo.http://www.cass.city.ac.uk/data/assets/pdf_file/0005/65174/PaolilloPetragallo.pdf

Rubio-Ramírez, J. F., Waggoner, J. F. D., \& Zha, T. (2005). Markov-switching structural vector autoregressions: Theory and application. Federal Reserve Bank of Atlanta, Working Paper 2005-27.

Sims, C., \& Zha, T. (1999). Error bands for impulse responses. Econometrica, 67, 1113-1155. https://doi.org/10.1111/1468-0262.00071

Sims, C., \& Zha, Z. (2006). Are there regime switches in US monetary policy? The American Economic Review, 96, 54-81.

Soledad, M., \& Martinez, P. (2002). A regime-switching approach to the study of speculative attacks: A focus on EMs crises. Empirical Economics, 27, 299-334. https://doi.org/10.1007/s001810100102

Valente, G. (2003). Monetary policy rules and regime shifts. Applied Financial Economics, 13, 525-36. https://doi.org/10.1080/0960310021000025001

\section{Appendix}

Figure 1A: Bivariate estimation of regime probabilities for inflation and monetary policy
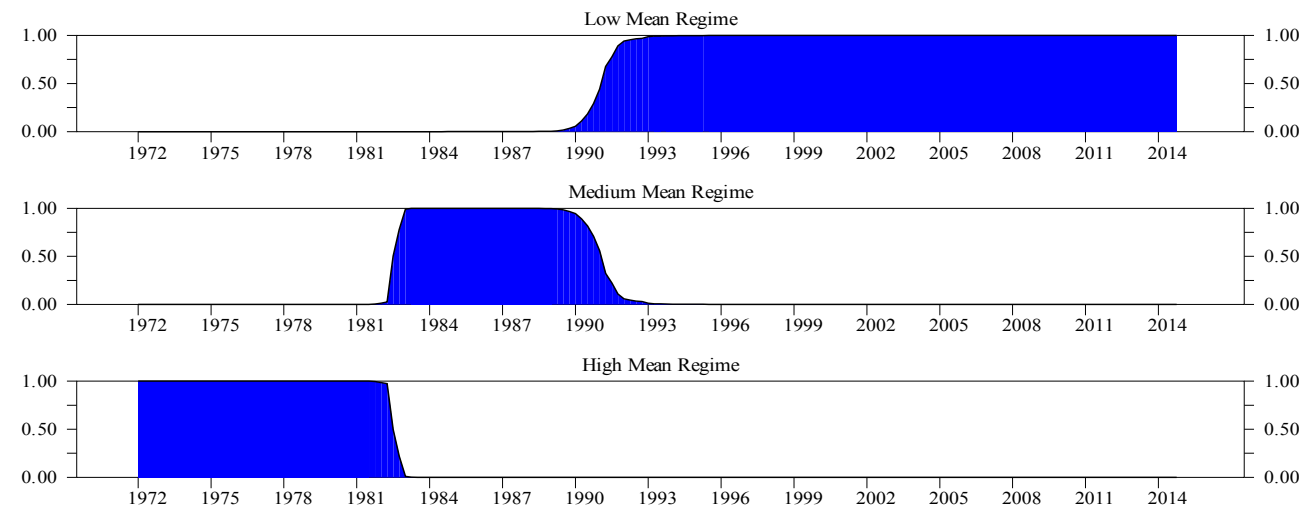

\section{Copyrights}

Copyright for this article is retained by the author(s), with first publication rights granted to the journal.

This is an open-access article distributed under the terms and conditions of the Creative Commons Attribution license which permits unrestricted use, distribution, and reproduction in any medium, provided the original work is properly cited. 